\title{
Dutch guideline for preventing nosocomial transmission of highly-resistant micro- organisms (HRMO) in long-term care facilities (LTCFs)
}

Andrea Eikelenboom-Boskamp ${ }^{1 *}$ D, Jobje Haaijman ${ }^{2}$, Maria Bos ${ }^{3}$, Katja Saris ${ }^{1}$, Else Poot ${ }^{4}$, Andreas Voss ${ }^{1,5}$ and On behalf of the expert group 'Long-term care facilities' of the former Dutch Working Party on infection control

\begin{abstract}
In 2012, the Dutch Working Party for Infection Control (WIP) issued the first Guideline for prevention of transmission of highly-resistant micro-organisms (HRMO) in Hospitals. The next step was to focus on long-term care facilities (LTCFs) both for nursing homes as for small-scale living facilities with nursing home care. These facilities providing care for residents with functional disabilities, chronical illnesses and cognitive disorders, such as dementia. The objective was to adapt the Guideline for prevention of transmission of HRMO in hospitals to LTCFs with a strong accent on living conditions and social interactions.

Residents of LTCFs may be carriers of HRMO over a long period of time and most of the residents of the LTCF stay for extended periods of time. To respect individual living circumstances and to prevent unnecessary limitations in the social life of the residents due to the use of isolation measures, the WIP has chosen to describe infection control precautions per individual micro-organism instead of a 'one size fits all' method. The term "isolation" was therefore replaced by the term "additional" precautions. This guideline describes the screening policies for residents in LTCFs, definition and detection of HRMO carriage, standard and additional infection control precautions for HRMO positive residents, documentation and communication of HRMO carriage and discontinuation of additional infection control precautions. It also describes contact tracing of HRMO, environmental control/investigation, surveillance of HRMO and what is important when there is an outbreak.
\end{abstract}

Keywords: Highly-resistant micro-organisms, HRMO, Antibiotic resistance, Antimicrobial resistance, Long-term care facilities, LTCF, Infection control precautions, Guidelines

\section{Introduction}

Antimicrobial resistance (AMR) is a worldwide threat to healthcare as common empiric antibiotics may no longer be effective to treat infections, including those that are life-threatening. Consequently, AMR may result in increased morbidity, mortality and cost of healthcare.

The World Health Organization (WHO) issued the Antimicrobial Resistance, Global Report on surveillance in 2014. This report summarizes all information on AMR and speaks of alarming levels of AMR in many

\footnotetext{
*Correspondence: eikelenboomandrea@gmail.com

${ }^{1}$ Canisius-Wilhelmina Hospital, Department of Medical Microbiology and

Infectious Diseases, Nijmegen, The Netherlands

Full list of author information is available at the end of the article
}

parts of the world [1]. In the Netherlands, AMR has, with a few exceptions, stayed on the same level from 2010 to 2015 [2]. Still, to control the increase in AMR, antibiotic should be used wisely and infection control precautions should be installed to prevent transmission of Highly Resistant Micro-Organisms (HRMO).

In 2012, the - by now former - Dutch Working Party for Infection Control (WIP) issued the first Guideline for prevention of transmission of HRMO in Hospitals [3, 4]. This guideline provides definitions for classification of HRMO and recommendations on surveillance, isolation precautions for patients and advice on outbreak management. It is currently implemented in Dutch hospitals. The next step was to focus on long-term care 
facilities (LTCFs), providing care for residents with functional disabilities, chronical illnesses and cognitive disorders, such as dementia. In the Netherlands, these residents are in the care of an "elderly care physician", a distinct medical specialization, exclusively working in LTCFs. Within the structure of the WIP, a so-called "Expert group LTCFs" was established, with professionals working (partially) in/for LTCFs. The expert group was tasked with the development of multiple Infection prevention and control guidelines, the first of which was 'the prevention of transmission of HRMO in LTCFs'.

Based on the Guideline for prevention of transmission of HRMO in hospitals, the ultimate goal was to adjust this guideline [5] to the living circumstances in LTCFs. This concerns all forms of nursing home care within institutions such as nursing homes or smallscale living facilities with the exception of geriatric departments of a hospital. These facilities have a strong emphasis on living conditions and social interactions. As residents of LTCFs may be carriers of HRMO over a long period of time [6] and as most of the residents of the LTCF stay for longer periods of time, infection control precautions may have a negative impact on the quality of life [7]. In order to respect individual living circumstances and to prevent unnecessary limitations in social life, the WIP has chosen to describe infection control precautions per individual micro-organism instead of a 'one size fits all' method. In addition, the term "isolation" was replaced by the term "additional precautions".

This guideline focusses on the control of HRMO and not for control of Methicillin-resistant Staphylococcus aureus (MRSA) for which a separate guideline is available in the Netherlands.

\section{Screening policies for residents in long-term care facilities}

When a resident is admitted to a LTCF and has stayed in a healthcare facility outside the Netherlands, HRMO screening must be initiated under the following circumstances:

- When the resident was admitted to a foreign health care facility (outside the Netherlands, the Caribbean islands not included) in the 2 months prior to admission to the LTCF and

- When the duration of admission in a foreign health care facility was longer than $24 \mathrm{~h}$

It is also advised to test a resident for HRMO carriage if the resident is transferred from a ward or small-scale living group within the healthcare facility or another healthcare facility with an ongoing HRMO outbreak within the Netherlands.

\section{Definition and detection of HRMO carriage}

The definition of HRMO is determined by the micro-organism and the specific antibiotic where the micro-organism has shown resistance to. The criteria for HRMO are based on the guideline "Laboratory detection of highly resistant microorganisms (HRMO)" of the Dutch society for Medical Microbiology [8]. This way, the definition of HRMOs are consistent with the established HRMO guideline for hospitals, making adequate information exchange easier $[3,4]$.

Three main groups of HRMO are distinguished: highly resistant Enterobacteriaceae (Table 1); highly resistant Gram-negative nonfermenters (Table 2), and highly resistant Gram-positive bacteria (Table 3).

To detect residents that carry HRMO, specific cultures have to be taken (Table 4).

\section{Standard and additional infection control precautions for HRMO positive residents}

In general, when giving physical care to residents, healthcare workers (HCWs) should always take standard precautions, such as adequate hand hygiene. These are meant to reduce the risk of transmission of pathogens from both known and unknown sources. The standard precautions are the minimal precautions a $\mathrm{HCW}$ must take in the care of all residents [9-11].

The additional infection control precautions are described in Table $5 \mathrm{a}$ and $\mathrm{b}$. In order to be clear and undisputable, all precautions are listed, including the standard precautions such as hand hygiene.

\section{Documentation and communication of HRMO carriage}

The documentation of the HRMO carriage is of utmost importance. Without knowing this, precautions to prevent transmission in the LTCF and other healthcare facilities (HCFs) cannot be taken. Therefore, all HCW involved, including those who are involved outside the LTCF (e.g. treating physicians in a hospital, primary care physicians), should be informed of the HRMO status of the resident. In addition, the HRMO status should be documented in the $(E)$ Health records for (para) medical and nursing staff.

Before transferring a HRMO positive resident to another ward/small-scale living group within the facility, or another facility, or before visiting e.g. an outpatient department, all those providing care should be informed about the HRMO status.

When a HRMO positive resident is re-admitted to a LTCF and there have not been 2 sets of negative HRMO cultures according to the rules mentioned in the section "Discontinuation of additional infection control precautions" below, additional precautions should be taken.

The HRMO carriers themselves/or the first contact person and their caregivers should be notified about 
Table 1 Definition of highly resistant Enterobacteriaceae

\begin{tabular}{lllll}
\hline Gram-negative rods & ESBL & Carbapenemase & Aminoglycosides & Quinolones \\
\hline Enterobacteriaceae & A & A & B & B \\
\hline
\end{tabular}

ESBL extended-spectrum beta-lactamase; A: presence of ESBL or Carbapenemase is sufficient to define the microorganism as highly resistant; $\mathrm{B}$ : resistance against both antibacterial agents from the two indicated groups is required to define the microorganism as highly resistant

the HRMO status in order to receive needed information with regard to the consequences as well as being able to apply adequate infection control measures. It is necessary that the physician-in-charge and other HCWs of the LTCF have the opportunity to consult a medical microbiologist and/or an infection control practitioner for advice regarding the prevention of transmission, diagnostics and treatment for HRMO positive residents.

\section{Discontinuation of additional infection control precautions}

Based on experience from earlier outbreaks and expert opinion, additional infection control precautions can be discontinued in the following cases:

- Resident, suspected for HRMO carriage:

$\circ$ If the HRMO screening cultures (Table 1) are negative, additional precautions can be discontinued. The resident should be without antibiotic treatment for at least $48 \mathrm{~h}$ before cultures are taken.

- Resident, HRMO positive

- If a resident is carrier of Enterobacteriaceae, (Extended-spectrum beta-lactamase (ESBL) included, Carbapenemase-producing Enterobacteriaceae (CPE) excluded), Acinetobacter species, Pseudomonas aeruginosa, Stenotrophomonas maltophilia and Streptococcus pneumonia (PRP), then additional precautions can be discontinued if at least 2 sets of HRMO screening cultures (taken at least $24 \mathrm{~h}$ apart) are negative.

- If a resident is carrier of CPE or vancomycinresistant Enterococcus faecium (VRE), additional precautions can be discontinued if at least 2 sets of cultures are negative, at least 1 year apart.

\section{Contact tracing of HRMO}

Contact tracing is recommended in case of unexpected HRMO positive residents. When a contact of the HRMO positive index appears to be HRMO positive too, it could be due to a single transmission event or it can be the result of broad transmission within a facility. In order to detect and prevent further transmission, contact tracing is recommended for all HRMO, possibly with an exception for ESBL positive Enterobacteriaceae or for Enterobacteriaceae resistant for Quinolones and Aminoglycosides. These two HRMOs are commonly found in the Dutch population with a prevalence of up to $8-10 \%$ in patients seeing a general practitioner [12].

The scale of the contact tracing is determined by the elderly care physician in collaboration with the medical microbiologist and/or infection control practitioner. In most cases, all residents who have been in contact with the HRMO positive resident will be cultured for HRMO carriage (see Table 4). Additional precautions can be postponed until the culture results from the first investigation are known. Directly changing precautions have much impact for the residents and HCWs and according to expert opinion is not advisable until transmission actually has been proven. If residents are transferred to another ward or HCF, it is advised to take additional precautions while waiting for culture results. Residents who are already discharged to their home-setting, will initially not be cultured unless in the first investigation HRMO positive residents are detected.

Contact tracing is also recommended if HRMO carriage is confirmed with a HRMO suspected resident and it is known that the additional precautions have not

Table 2 Definition of highly resistant gram-negative nonfermenters

\begin{tabular}{|c|c|c|c|c|c|c|}
\hline $\begin{array}{l}\text { Gram-negative } \\
\text { nonfermenters }\end{array}$ & Carbapenemase & Aminoglycosides & Quinolones & Ceftazidime & Piperacillin & Co-trimoxazole \\
\hline Acinetobacter spp. & $A$ & B & $B^{a}$ & $\mathrm{n} / \mathrm{a}$ & $\mathrm{n} / \mathrm{a}$ & $\mathrm{n} / \mathrm{a}$ \\
\hline $\begin{array}{l}\text { Stenotrophomonas } \\
\text { maltophilia }\end{array}$ & $\mathrm{n} / \mathrm{a}$ & $\mathrm{n} / \mathrm{a}$ & $\mathrm{n} / \mathrm{a}$ & $\mathrm{n} / \mathrm{a}$ & $\mathrm{n} / \mathrm{a}$ & A \\
\hline $\begin{array}{l}\text { Pseudomonas } \\
\text { aeruginosa }\end{array}$ & C & C & C & C & C & $\mathrm{n} / \mathrm{a}$ \\
\hline
\end{tabular}

anly Ciprofloxacin and/or Levofloxacin, due to the intrinsic resistance of Acinetobacter species for norfloxacin; A: Carbapenemase or resistance against an antibacterial agent from the indicated group is sufficient to define the microorganism as highly resistant; B: resistance against antibacterial agents from at least two indicated groups is required to define the microorganism as highly resistant; C: resistance against antibacterial agents from at least three of the indicated groups is required to define the microorganism as highly resistant; n/a: not applicable 
Table 3 Definition of highly resistant gram-positive bacteria ${ }^{a}$

\begin{tabular}{lll}
\hline Gram-positive bacteria & Penicillins & Vancomycin \\
\hline Streptococcus pneumoniae & A & A \\
Enterococcus faecium & B & B
\end{tabular}

${ }^{a}$ : MRSA not included; A: resistance against an antibacterial agent from the indicated group is sufficient to define the micro-organism as highly resistant; $B$ : resistance against both antibacterial agents from the two indicated groups is required to define the microorganism as highly resistant

adequately been performed in the time between culturing and results. In that time HRMO transmission could have taken place.

Contact tracing among HCWs is not indicated. HCW, if at all, are only transient carriers of HRMO. In addition - and in contrast to MRSA - possibilities for decolonization treatment of HRMOs is limited and not routinely used.

\section{Environmental control/investigation}

Initially, culturing the environment to detect a source of the HRMO is not indicated. If, however, during an outbreak with HRMO transmission persists, environmental culturing may be considered to determine a source of the outbreak.

\section{Surveillance of HRMO}

Evaluation of the local and regional epidemiology of HRMO provides knowledge in the, sometimes rapidly changing, evolution in this area.

Performing surveillance on a local and regional level, by exchanging on a regular basis HRMO data from routine diagnostics, can be very helpful to determine if there is an indication of an increasing level of HRMO. At present, gathering and combining data to receive insight into the regional epidemiology is a task for the Dutch regional networks, initiated by the ministry of health, to combat AMR. To have unbiased surveillance data (at least once a year) point-prevalence studies among the residents of the LTCF should be performed by trained professionals to determine local levels and possible transmission of HRMO in the facility.

\section{Outbreak}

A situation is considered to be an outbreak when 2 or more residents have the same HRMO and the presence of an epidemiological link between them.

During an outbreak, it is important to maintain and highlight the standard precautions and additional precautions specific for that kind of HRMO (as described in Table 5b).

It is strongly advisable to install an Outbreak Management Team. This multidisciplinary team consists an elderly care physician, medical microbiologist, infection control practitioner, staff members of the wards involved (both nursing and medical), member of the management team and professional of the Local Health Authority.

This team which will take care of the arising issues such as decisions on additional infection control precautions, adjustment of antibiotic therapy, communication within and outside of the LTCF where the outbreak takes place and alert national authorities of this specific outbreak.

When despite tightening up infection prevention precautions, further transmission takes place, confirmation of clonal relationship between the strains or plasmids by molecular typing needs to be done [13].

\section{Discussion}

There are limitations to this guideline for HMRO carriage in long term care settings.

Table 4 Diagnostic screening procedure for residents suspected for HRMO carriage in LTCF

\begin{tabular}{|c|c|c|}
\hline Micro-organism/Indication & Standard Cultures ${ }^{a}$ & Additional cultures (when indicated) ${ }^{a, b}$ \\
\hline Enterobacteriaceae (ESBL and CPE inclusive) & Rectal swab or stool sample & Wound swab, sputum sample, urine sample \\
\hline Acinetobacter species & $\begin{array}{l}\text { Rectal swab or stool sample and sputum } \\
\text { sample or oropharyngeal swabc }\end{array}$ & Wound swab, urine sample \\
\hline Stenotrophomonas maltophilia & $\begin{array}{l}\text { Rectal swab or stool sample and sputum } \\
\text { sample or oropharyngeal swabc }\end{array}$ & Wound swab, urine sample \\
\hline Pseudomonas aeruginosa & $\begin{array}{l}\text { Rectal swab, stool sample and sputum } \\
\text { sample or oropharyngeal swabc }\end{array}$ & Wound swab, urine sample \\
\hline Streptococcus pneumoniae & Sputum sample or oropharyngeal swabc & - \\
\hline Enterococcus faecium & Rectal swabs or stool samples & Wound swabs, sputum samples, urine samples \\
\hline $\begin{array}{l}\text { When resident is transferred from health care } \\
\text { facility outside the Netherlands }\end{array}$ & $\begin{array}{l}\text { Rectal swab or stool sample and sputum } \\
\text { sample or oropharyngeal swabc }\end{array}$ & Wound swab, sputum sample, urine sample \\
\hline
\end{tabular}

${ }^{\mathrm{a}}$ Single swab/sample from the stated site, excepting for Enterococcus faecium. Standard and additional cultures for Enterococcus faecium: five swabs/samples on five consecutive days

${ }^{\mathrm{b}}$ Depending on clinical presentation of the signs and symptoms of resident: - culture of sputum when resident has a persistent cough - culture of wound if present

- urine culture when urinary tract catheter is in place

c Preferably sputum sample. If sputum sample cannot be obtained, collect oropharyngeal swab 


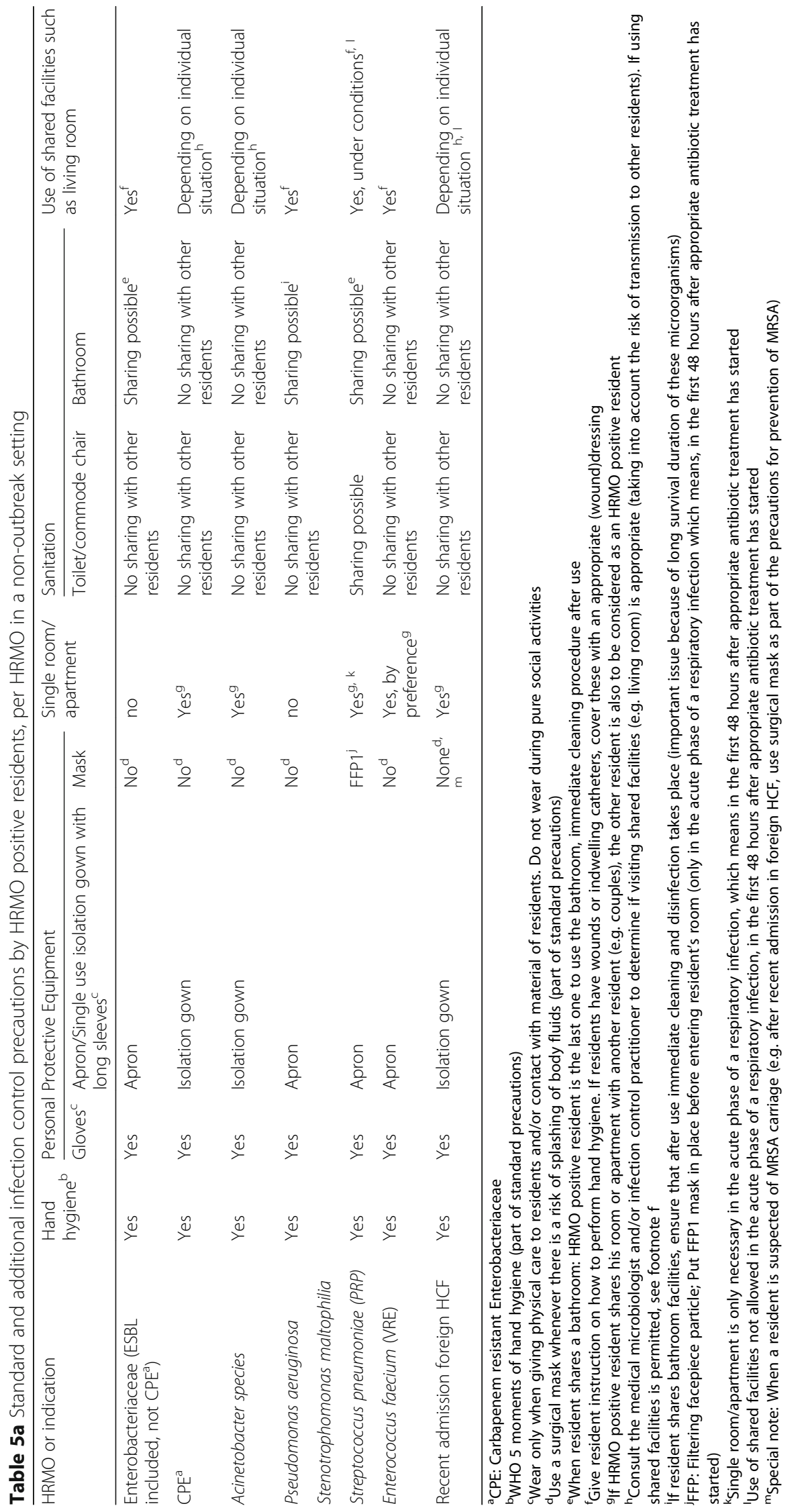




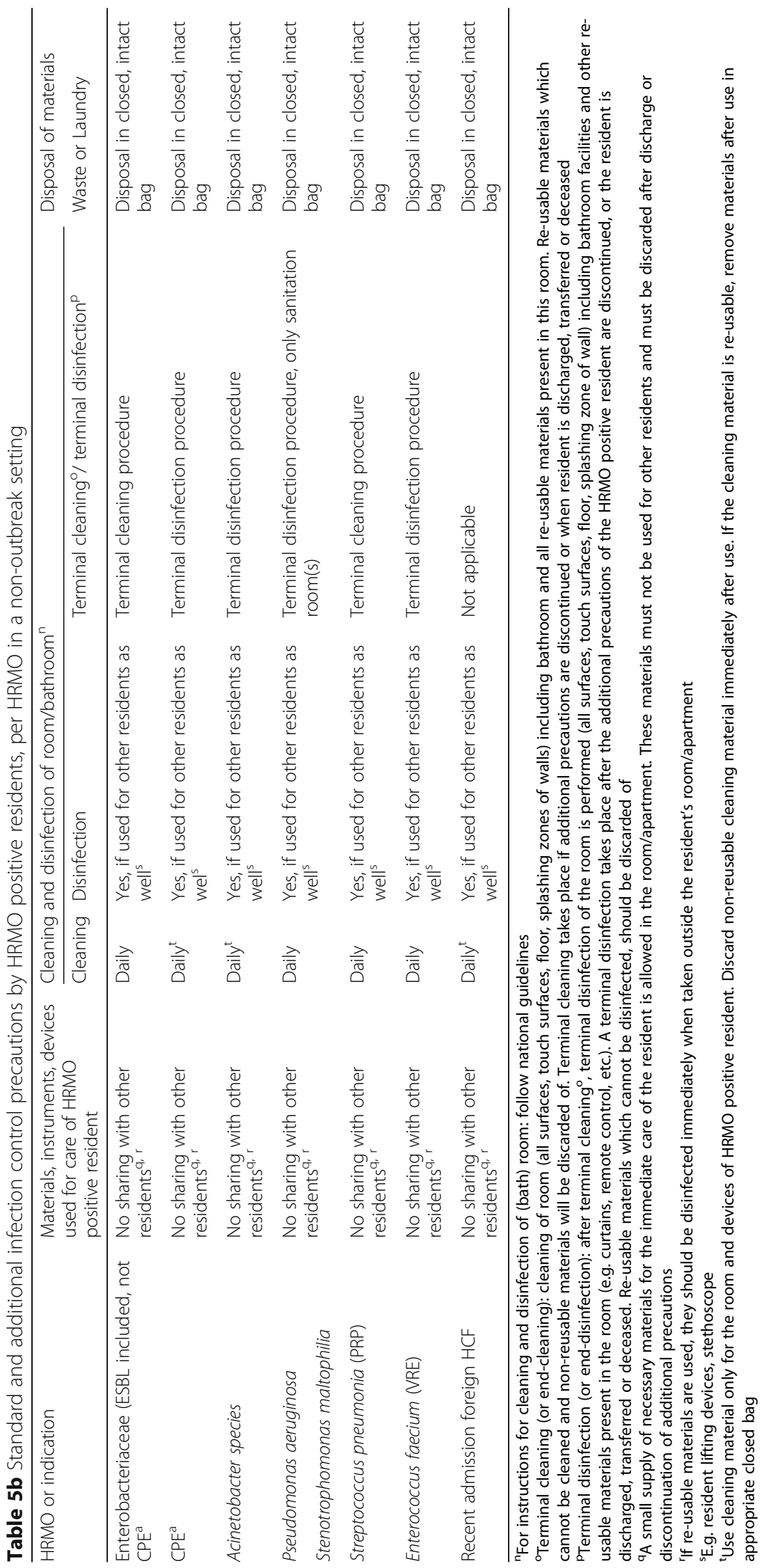


First, the absolute risk of transmission of HRMO within the Dutch LTCFs (as defined in the guideline) is not known. However, there is a growing understanding of the potential for transmission of HRMO in the LTCF. In 2016, den Dool et al. used mathematical modelling to estimate the contribution of nursing homes in the dispersal of pathogens over the healthcare network in the Netherlands. They concluded that nursing homes have the potential to drive and sustain epidemics across this network and that infection control efforts and surveillance systems should also be targeted at those LTCFs [14]. Recent research in Dutch LTCFs showed that, although in absolute numbers the percentage of HRMO is low (4.2\% Escherichia coli ESBL carriage among residents), the large variation of HRMO presence between facilities (1-33\%) warrants cautious surveillance [15].

Secondly, it is not known how long a resident remains colonised with HRMO. Research shows that carriage can persist over years, depending on the micro-organism [15]. The guidance for the decision to discontinue infection control precautions is therefore based on expert opinion. Although research indicates that there might be predisposing factors for prolonged carriage, more research is needed to determine when to discontinue precautions and consider HRMO carriage as ended in longterm care. Despite the lack of studies that show the effect of monitoring of the HRMO carriage of a resident and its consequences in LTCFs, it is logical to assume that these measures are effective to prevent the transmission of HRMO.

Last but not least, it is not known whether the proposed actions in the LTCF on prevention of transmission of HRMO, are equally effective and achievable for the various groups of residents in such facilities, such as e.g. psychogeriatric residents. However, given the rising evidence for spreading of HRMO within the LTCF settings, this is a first step in developing guidelines for prevention of transmission of HRMO. Over the course of time, with leaders in both infection control and LTCFs, further guidance should be provided, while the absolute risk of transmission and harm as opposed to the adverse events related to additional precautions, such as reduced psychological wellbeing, resident safety and satisfaction in residential care [7].

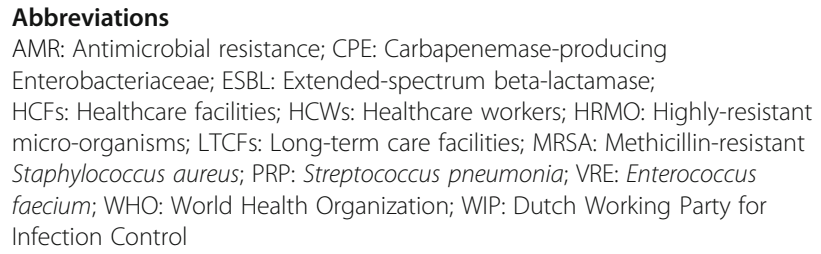

\section{Acknowledgements}

We thank the members of the Dutch Working Party Expert group (WIP) for infection control in long-term care facilities: Dr. E. Stobberingh, Drs. E.
Gorissen-Douven, P. Molenaar, R. Hoentjen ${ }^{\dagger}$,Drs. P. Tolsma, I. Verzijl and other members of this group. We also thank the members of the coordination group of the WIP.

Recently, the WIP was dissolved, to be replaced by a new broadly-based guideline structure, including subgroups for medical care, public-health and long-term care. The new 'network for infection control guidelines (SRI)' needs still to be established.

\section{Authors' contributions}

$A E-B, J H$ and $M B$ contributed to writing the manuscript. KS, EP and AV read, commented and approved the final manuscript.

\section{Funding}

Not applicable.

Availability of data and materials

Not applicable.

Ethics approval and consent to participate

Not applicable.

\section{Consent for publication}

All named authors have seen and agreed the submitted version of the paper. All persons who are included in the acknowledgements section have agreed to that inclusion.

\section{Competing interests}

The authors declare no conflict of interest.

\section{Author details}

${ }^{1}$ Canisius-Wilhelmina Hospital, Department of Medical Microbiology and Infectious Diseases, Nijmegen, The Netherlands. ${ }^{2}$ River Region Elderly Care Center (SZR), Tiel, The Netherlands. ${ }^{3}$ Avans University of Applied Sciences, Breda, The Netherlands. ${ }^{4}$ Verenso Dutch Association of Elderly Care Physicians and Social Geriatricians, Utrecht, The Netherlands. ${ }^{5}$ Radboud University Medical Centre, Department of Medical Microbiology, Nijmegen, The Netherlands.

Received: 9 April 2019 Accepted: 28 July 2019

Published online: 27 August 2019

\section{References}

1. World Health Organization (WHO). Antimicrobial resistance: global report on surveillance 2014. Www.WHO.int.

2. The Dutch working party on antibiotic policy (SWAB). NethMap 2015: consumption of antimicrobial agents and antimicrobial resistance among medically important bacteria in the Netherlands. www.swab.nl.

3. Dutch working party on infection prevention. Policy for highly resistant micro-organisms (HRMO) hospitals. 2012. Www.wip.nl.

4. Kluytmans-Vandenbergh MF, Kluytmans JA, Voss A. Dutch guideline for preventing nosocomial transmission of highly resistant microorganisms (HRMO). 2005;33(5-6):309-313.

5. Dutch working party on infection prevention. Policy for highly resistant micro-organisms (HRMO) long term care facilities. 2014. Www.wip.nl.

6. Kola A, Holst M, Chaberny IF, Ziesing S, Suerbaum S, Gastmeier P. Surveillance of Extendend-spectrum Beta-lactamase producing bacteria and routine use of contact isolation: experience of a three-year period. J Hosp Infect. 2007;66(1). https://doi.org/10.1016/j.jhin.2007.01.006.

7. Abad C, Fearday A, Safdar N. Adverse effects of isolation in hospitalised patients: a systematic review. J Hosp Infect. 2010;76(2):97-102.

8. Bernards et al. Laboratory detection of highly resistant micro-organisms. NVMM (Dutch Society for Medical Microbiology). Www.nvmm.nl.

9. World Health Organization (WHO). WHO guidelines on hand hygiene in health care. Www.WHO.int

10. Dutch Working Party on Infection Prevention. Policy for personal hygiene healthcare workers and volunteers in long term care facilities. 2017. Www. wip.nl.

11. Dutch Working Party on Infection Prevention. Policy for personal protect equipment for healthcare workers in long term care facilities. 2014. Www.wip.nl.

12. Reuland EA, Overdevest IT, AI Naiemi N, Kalpoe JS, Rijnsburger MC, Raadsen $\mathrm{SA}$, et al. High prevalence of ESBL-producing Enterobacteriaceae carriage in 
Dutch community patients with gastrointestinal complaints. Clin Microbiol Infect. 2013;19(6):542-9. https://doi.org/10.1111/j.1469-0691.2012.03947.x.

13. Willemsen I, Elberts S, Verhulst C, Rijnsburger M, Filius M, Savelkoul P, et al. Highly resistant gram-negative microorganisms: incidence density and occurrence of nosocomial transmission (TRIANGLe study). Infect Control Hosp Epidemiol. 2011;32(4):333-41. https://doi.org/10.1086/658941.

14. Van den Dool C, Haenen A, Leenstra T, Wallinga J. The role of the nursing homes in the spread of antimicrobial resistance over the healthcare network. Infect Control Hosp Epidemiol. 2016;37(7) 10.1017\%2Fice.2016.59.

15. Verhoef L, Roukens M, de Greeff S, Meessen N, Natsch S, Stobberingh E. Carriage of antimicrobial-resistant commensal bacteria in Dutch long-term care facilities. J Antimicrob Chemother. 2016;71(9):2586-92. https://doi.org/10.1093/jac/dkw183.

\section{Publisher's Note}

Springer Nature remains neutral with regard to jurisdictional claims in published maps and institutional affiliations.

Ready to submit your research? Choose BMC and benefit from:

- fast, convenient online submission

- thorough peer review by experienced researchers in your field

- rapid publication on acceptance

- support for research data, including large and complex data types

- gold Open Access which fosters wider collaboration and increased citations

- maximum visibility for your research: over $100 \mathrm{M}$ website views per year

At BMC, research is always in progress.

Learn more biomedcentral.com/submissions 